\title{
Using DEM Data Simulation of SAR Image Based on Range-Doppler Model
}

\author{
Dongjian Xue ${ }^{1,2,3, *}$, Jie Zheng ${ }^{1,3}$, Wanqiu $\mathrm{Li}^{1,3}$, Lulu Su${ }^{1,3}$ and Chengrao $\mathrm{Li}^{1,3}$ \\ ${ }^{1}$ College of Earth Sciences of Chengdu University of Technology, Chengdu Sichuan 610059, China \\ ${ }^{2}$ Research Institute of Forest Resources Information Techniques, Chinese Academy of Forestry, Beijing 100091, China \\ ${ }^{3}$ Key Laboratory of Geoscience Spatial Information Technology, Ministry of Land and Resources of the P.R. China, 610059 \\ ${ }^{*}$ Corresponding author
}

\begin{abstract}
Based on the analysis of SAR imaging geometry and characteristics of satellite orbit parameters, This article mainly introduces $R D$ location model, radar equation, backscattering model, principle of simulating SAR image and so on. The Range Doppler $(R D)$ geo-location model is used to calculate the position of the radar imaging point for each grid point of the DEM. The SAR image is simulated and extracts the local incidence angle, projection angle and so on. The simulated SAR image coincides with the real SAR image texture through simulation experiment, which is beneficial to the automatic registration of the control points, especially in the mountains, where is very difficult to get the ground control point.
\end{abstract}

Keywords- PALSAR; digital elevation model; SAR simulation; range-doppler model

\section{INTRODUCTION}

Synthetic Aperture Radar (SAR) can work all weather and all day.SAR has certain penetration on the surface, which determines it has great advantages and application prospect compared to optical image. However, it is difficult to deal with SAR because of its unique imaging geometry. SAR image simulation is a way to study the characteristics of SAR image, which is based on the actual surface features, SAR imaging parameters and so on ${ }^{[1,2]}$.The use of simulated SAR image helps image correction, radar system optimization, Interferometric, and interpretation of auxiliary SAR images. The iterative optimization of the model parameters in the calibration process requires the use of control points. In the past, using artificial acquisition the control points can take a lot of manpower and it is very difficult to obtain the high precisions in the mountain area. It has a greater advantage to automatic registering the same feature points on simulation SAR images and real SAR images by using image matching technology. At present, the simulation methods of SAR images, mainly divided into three categories: coherent system simulation, non coherent image simulation and simulation based image. In this paper, according to the SAR imaging parameters, the mapping relationship between the ground coordinate system and the oblique coordinate system, simulation image is established by using $R D$ location model based on DEM.

\section{SAR SYSTEM PARAMETER AND GEOMETRIC POSITIONING MODEL}

\section{A. Introduction of the ALOS PALSAR System Parameters}

ALOS satellite is Japan's earth observation satellite, orbital height $691.65 \mathrm{~km}$, cycle $98.7 \mathrm{~min}$ and repeat cycle 46 days with three sensors. And the sensors PALSAR has three kinds of observation modes: high resolution mode, scanning synthetic aperture radar and polarization mode. And the high resolution mode can divided into fine beam single polarization mode (FBS) and fine beam dual polarization mode(FBD). The data in this paper is from ALOS PALSAR image taken in September 11, 2010(Table I), and the study area is located at the binding site of Anxian and Maoxian in Sichuan, its geographic coordinates are East longitude $103^{\circ} 35^{\prime} 47^{\prime \prime} \sim 104^{\circ} 22^{\prime} 40^{\prime \prime}$, and North latitude $31^{\circ} 23^{\prime} 48^{\prime \prime} \sim 32^{\circ} 00^{\prime} 13$. The area has diverse terrain, mainly for hilly, flat topography,middle and lower mountain and so on. That is to take Daguangbao oblique fault and Beichuan thrust fault as a boundary. The northwestern part of the boundary belongs to western Sichuan geosyncline zone of the Longmen mountain fold belt which is longmen mountains with higher terrain. Daguangbao in Gaochuan country is 3047 meters above sea level is the highest and the lowest 490 meters above sea level is located at Anchang river, the junction of Shiancun village in Jiepan town and Mianyang city.

TABLE I. PARAMETERS OF ALOS PALSAR IMAGES

\begin{tabular}{|c|c|c|c|c|c|c|c|}
\hline \multirow{2}{*}{ Imaging time } & \multicolumn{2}{|c|}{ Center longitude and latitude ( ${ }^{\circ}$ ) } & \multirow{2}{*}{$\begin{array}{c}\text { Incidence_angle } \\
\left({ }^{\circ}\right)\end{array}$} & \multirow{2}{*}{$\begin{array}{c}\text { Near range } \\
(\mathrm{m})\end{array}$} & \multicolumn{2}{|c|}{ Resolution ( m) } & \multirow{2}{*}{ Pol } \\
\hline & Latitude & Longitude & & & Range & Azimuth & \\
\hline 20100911 & 31.6937 & 103.9823 & 38.7199 & 846567 & 9.368514 & 3.166026 & $\mathrm{HH}$ \\
\hline Wavelength (m) & \multicolumn{2}{|c|}{ Prf(HZ) } & $\begin{array}{c}\text { Azimuth } \\
\text { bandwidth (HZ) }\end{array}$ & \multicolumn{2}{|c|}{ Chirp Bandwidth(MHZ) } & $\begin{array}{l}\text { Azimuth } \\
\text { lines }\end{array}$ & $\begin{array}{c}\text { Range } \\
\text { samples }\end{array}$ \\
\hline 0.236 & \multicolumn{2}{|c|}{2150.54} & 1523.25 & \multicolumn{2}{|c|}{14} & 18432 & 4640 \\
\hline
\end{tabular}




\section{B. Geometric Positioning Model of SAR}

As an active remote sensing imaging mode, SAR provides the distance and the Doppler history information between the sensor and the target, and these information can link the satellite to the earth's surface accurately, thus we can construct the SAR positioning model and get geographic coordinates of each pixel by resolving the model. The $R-D$ model was first proposed by Brown (W.E.Brown), developed by Curlander et al. (1982,1991),the latter gave three basic equations as the starting point of the problem- solving. The principle of $R-D$ positioning is to determine the position of the pixel by using the intersection of equidistant line and equal Doppler line on the contour plane of the earth (Figure I).

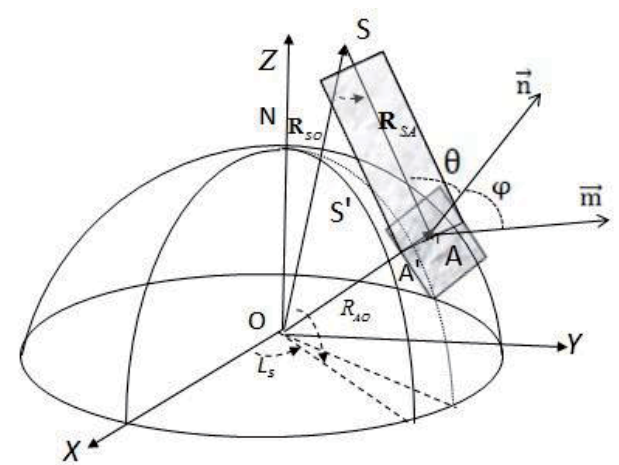

FIGURE I. SCHEMATIC DIAGRAM OF SAR IMAGING GEOMETRY STRUCTURE

In Figure I, $S$ represents the position of the satellite. $A$ is a feature point on the surface of the Earth, the Projection Point of the feature $A$ on the surface of the ellipsoid is $A$ ',the $h$ is height of the ground point $A A$ ', the position and velocity vector of the satellite respectively are $R_{S O}$ and $V_{S O}$, and the position vector and velocity vector of point $A$ are $R_{A O}$ and $V_{A O}$.The geocentric inertial coordinate system (GEI) coordinate vector of feature point $A$ is $R_{A O}=\left[\begin{array}{lll}X & Y & Z\end{array}\right]^{T}$.For WGS-84 ellipsoid is

$R_{a}=637813 \mathrm{~m}$ and $R_{b}=6356752 . \mathrm{m} R$ is the distance from the satellite to the target. $f_{D}$ is $A$ point corresponding to the doppler center frequency, ${ }^{\lambda}$ is radar wavelength, and $R$ is the distance of satellite and ground point in A point imaging time. The $R-D$ localization model establishes the relationship between the image points $(x, y)$ and the features $(X, Y, Z)$ from the geometrical perspective of SAR imaging. The resulting equation: equation (1) is the ellipsoid equation which is $(X, Y, Z)$ to be satisfied. equation (2) is the distance equation of the satellite position vector and the target vector. equation (3) is the doppler shift frequency equation. The location of each cell can be attained by solving. The parameters for resolving the $\mathrm{RD}$ positioning model can be extracted from the PALSAR header file.

$$
\begin{gathered}
\frac{X^{2}+Y^{2}}{\left(R_{a}+h\right)^{2}}+\frac{Z^{2}}{R_{\mathrm{b}}^{2}}=1 \\
R^{2}=\left(X-X_{S}\right)^{2}+\left(Y-Y_{S}\right)^{2}+\left(Z-Z_{S}\right)^{2} \\
f_{\mathrm{D}}=-\frac{2}{\lambda} \frac{\left(\mathbf{R}_{S O}-\mathbf{R}_{A O}\right) \cdot\left(\mathbf{V}_{S O}-\mathbf{V}_{A O}\right)}{R}
\end{gathered}
$$

\section{BACKSCATTERING MODEL}

Radar image simulation is based on the actual situation on the ground or orbital data. According to the radar image imaging mechanism, in accordance with the point scattering model, applying radar imaging geometry formula, radar equation and gray equation, generating a radar image by the computer.

$$
P_{r}=\frac{P_{t} G^{2}(\theta) \lambda}{(4 \pi)^{3} R^{4}} \sigma
$$

When the SAR image is simulated, the ground be divided into the grid based on the size of the resolution unit. Each grid is a ground element corresponding to a pixel of the radar image. Simulation of radar image is to make surface features in every grid on the ground form a radar image, according to the scattering characteristics of ground objects and imaging principle, in term of equation (4) and gray equation, calculation of scattering intensity of each piece of ground units, and then converted into the gray value of the image. In the radar equation, the calculation of $\sigma$ is more complicated, and it is related to wavelength, polarization mode, local incidence angle, complex permittivity, vegetation cover, surface roughness and so on, and some parameters are difficult to be obtained accurately, The backscattering energy is difficult to calculate, often using physical models or empirical formulas to describe the backscattering coefficient, such as a backscatter model is proposed by Lu Zhang ${ }^{[10]}$ which consider the area of radar cross-section is related to the parameters of radar system.

$$
A=\delta_{\mathrm{a}} *\left|\frac{\mathrm{ga}_{\mathrm{a}}+\delta_{\mathrm{r}} * \cos \beta}{\sin \beta}\right|
$$

$\delta_{\mathrm{a}}$ and $\delta_{\mathrm{r}}$ represent azimuth and distance to the resolution respectively, ${ }^{{ }_{a}}$ represents the rate of change in elevation from the direction, and $\beta$ is an incident angle.

Muhleman proposed a semi-empirical backscattering model (6) which can calculate the radar cross section (Radar Cross Section-RCS) $\sigma$.

$\sigma$ is the radar cross section and $\theta$ is the local incident angle. $\beta$ and ${ }^{\theta}$ can be settled with reference to the figure. In Figure I, $\overrightarrow{\mathrm{n}}$ is the normal of the ground unit, $\overrightarrow{\mathrm{m}}$ is the normal of the imaging plane, ${ }^{\theta}$ is the local incident angle (the angle between $\overrightarrow{\mathrm{n}}$ and the incident vector). $\beta$ is the reference incidence angle.

$$
\begin{aligned}
& \theta=\arccos \left(\frac{\overrightarrow{\mathrm{n}} \cdot \mathrm{R}_{\mathrm{SA}}}{\left|\overrightarrow{\mathrm{R}_{\mathrm{SA}}}\right| \cdot|\overrightarrow{\mathrm{n}}|}\right) \\
& \beta=\arcsin \left(\frac{\left|R_{S O}\right|}{\left|R_{A 0}\right|} * \sin \gamma\right)
\end{aligned}
$$

The SAR image coordinates $(i, j)$ and the satellite position vector $R_{S A}$ are determined by the SAR satellite imaging parameters and the established R-D positioning model. The $R_{A O}$ and $^{\overrightarrow{\mathbf{n}}}$ can be calculated by the known DEM. 
As the contribution of $\sqrt{\sigma}$ and DEM resolution unit to the scattering energy of the simulated SAR image is proportional, Wivell, C.E ${ }^{[11]}$ uses $k \cdot \sqrt{\sigma}$ as the simulation image value of the pixel, $\mathrm{k}$ is a constant:

$$
D N=k \cdot \sqrt{\sigma}
$$

Guindon thinked that the characteristics of the simulated image are determined by the sum of the actual ground scattering unit area. It is not important to calculate what kind of backscattering modelo is used. He suggested that the above equation can be defined as:

$$
D N=K
$$

Where $\mathrm{K}$ is an arbitrary constant, then the backscatter model is purely mathematical model, and no physical meaning.

\section{SAR IMAGE SIMULATION}

In this paper, the mapping of ground coordinates $P(X, Y, Z)$ to the $(x, y)$ coordinates of the imaging point SAR image is established by using DEM. Because the resolution of DEM is lower than that of SAR, in order to improve the continuity of analog SAR image pixels, the simulated SAR image has better visual effect. Firstly, the DEM is oversampled. According to the PALSAR imaging parameters, the $R D$ location model is used to compute the SAR image coordinates $(x, y)$ of each pixel of the sampled DEM and save the result to the lookup table (LUTA). According to the simulated SAR image, The local incidence angle of the pixel (Figure II) and the projection angle. According to the texture and geometric features, the SAR image is automatically registered with the SAR to be corrected, and the polynomial equation is established. The real SAR image coordinates $(x, y)$ corresponding to the SAR image coordinates $(x, y)$ are calculated and updated (LUTA), calculate the true SAR image coordinates $(x, y)$ corresponding to each DEM pixel, and resample the pixel value into the DEM coordinate space to obtain the analog SAR image with the same size as the DEM (Figure III).

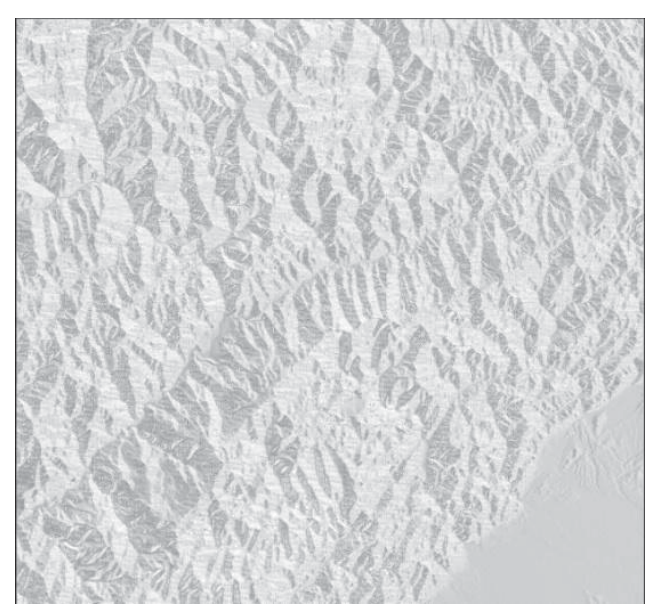

FIGURE II. LOCAL ANGLE OF INCIDENCE

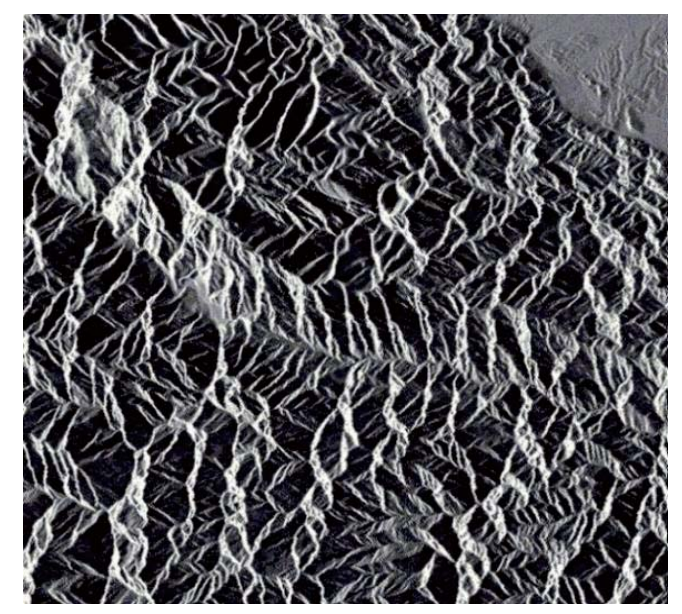

FIGURE III. SIMULATED SAR IMAGE BASED ON DEM

It reflect the basic features of the imaging such as contraction, overlapping, shadow that the simulated image texture features are close to the real SAR image. Since the backscatter intensity of the simulated image is calculated by multiplying the empirical function of the pixel and the local incidence angle, because the independence of the surface backscatter is not taken into account at the time of simulation, it is constant for most of the plain area. Most of them are mountainous, and do not affect the automatic registration of the whole image.

\section{CONCLUSION}

It is very important to use the DEM to simulate the SAR image because of the special imaging mode of the radar. Taking the ALOS PARSAR data as an example, a SAR image simulation method based on DEM is mainly studied on the basis of analyzing SAR imaging geometry and backscatter model. The simulated SAR image is very close to the actual SAR image in the mountainous area, which can reflect the terrain feature of the mountain area and is helpful for the automatic registration of the control point.

\section{ACKNOWLEDGMENT}

Key Fund Project of Key Laboratory of Geoscience Spatial Information Technology, Ministry of Land and Resources of the P.R.China(KLGSIT2013-06),Key Project of Sichuan Provincial Department of Education(16ZA0100).

\section{REFERENCES}

[1] Loew A,Mauser W.Generation of geometrically and radiometrically terrain corrected SAR image products[J].Remote sensing of Environment,2007,106(3):337-349.

[2] Shu ning.Principles of Microwave Remote Sensing[M],2001.

[3] CHEN Er xue, LI Zengyuan,etc.Terrain Radiometric Correction Model and Its Validation for Space-Borne SARData[J].Geomatics and Informat ion Science of Wuhan Univer sity, 2010,35(3):322-327.

[4] Shimada M.Ortho-Rectification and Slope Correction of SAR Data Using DEM and Its Accuracy Evaluation[J].IEEE Journal of Selected Topics in Applied Earth Observations and Remote Sensing,2010,3(4):657-671. 
[5] WU Tao,WANG Chao,ZHANG Hong, Space-borne SAR Image Smiulation Based on Image Characteristics[J]. JOURNAL OF REMOTE SENSING,2005,(4):237-240.

[6] Leberl,F,Radargrammetric Image Processing,Artech House, Norwood,Massachusetts.,1990

[7] Franceschetti G,Migliaccio M,Riccio D.SARAS:A Synthetic Aperture Radar(SAR)Raw Signal Simulator[C],IEEE Transactions on Geoscience and Remote Sensing,1992,30(1):110-123.

[8] Dongjian Xue,Jie Zheng,Chengrao Li,etc.Landslides Monitoring with SAR/InSAR Technolog[J],The third imaging radar senior academic seminar of earth observation.2016:542-545.

[9] QIN Zhi-yuan, ZHANG Bao-yin,etc.Simulation of SAR Images Based on DEM and Classification Information of Ground Objects[J]. Journal of System Simulation,2009(08):2437-2440.

[10] Zhang L,Balz T,Liao MS.Satellite SAR geocoding with refined RPC mode[J].ISPRS Journal of Photogrammetry and Remote Sensing,2012,69:37 49.

[11] Wivell,C.E.,Steinwand,D.R,Kelly, G.G, Meyer,D.J.,1992, Evaluation of Terrain Models for the Geocoding and Terrain Correction of Synthetic Aperture Radar (SAR) Images, IEEE. Trans. Geosci. Remote Sensing,vol.30, no.6, pp.1137 1144.

[12] Muhleman D O.Radar scattering from Venus and Moon[J].Astronomic Journal.1964(24):34-40.

[13] SMALL D F Lattening Gamma:Radiometric Terrain Correction for SAR Imagery[J].IEEE Transactions on Geoscience and Remote Sensing,2011,49(8):3081-3093. 